\title{
A case of palmoplantar pustulosis complicated with vitiligo vulgaris
}

\section{Mayu Sato, Toshiyuki Yamamoto}

\author{
Department of Dermatology, Fukushima Medical University, Fukushima, Japan
}

Corresponding author: Dr. Mayu Sato, E-mail: s-mayu@fmu.ac.jp

Sir

Both vitiligo vulgaris and palmoplantar pustulosis have been suggested to associate with autoimmune pathogenesis. We here report a case of concurrent occurrence of vitiligo and palmoplantar pustulosis in a same patient.

A 44-year-old female was referred to our department, complaining of small vesicles and pustules on the soles of both feet, which had appeared approximately 6 months previously. Arthralgia had also appeared about 3 months prior to presentation. On physical examination, erythema, scales, and small vesicle and on the left sole were observed (Fig. la), and small pustules were also scattered close to the erythema (Fig. 1b). Palms were not involved. Joint pain accompanied in the region of left cervix, clavicle, and shoulder. Furthermore, a depigmented macule surrounded by slight hyperpigmentation was also found on the right back side of the neck (Fig. 2). Laboratory data on blood chemistry, including liver and renal function, were within the normal ranges. Antinuclear antibody, and anti-thyroglobulin antibodies were negative. However, anti-thyroid microsomal antibodies were slightly positive (1:100). Bone scintigraphy showed increased uptake of technetium-99m in the left sternoclavicular joint (Fig. 3). We searched for infected foci, and CT of the paranasal sinuses revealed a small amount of soft tissue shadow in both the ethmoid sinus and maxillary sinus. No bone destruction was observed, and a diagnosis of chronic sinusitis was made. In the dental examination, there was a percussive pain in the upper left molar, and X-ray confirmed inflammation in the apical part of the tooth; therefore, a diagnosis of apical periodontitis was made.
The present case developed palmoplantar pustulosis, pustulotic arthro-osteitis, and vitiligo. Vitiliginous lesion was observed only on the nape. Several studies suggest that autoimmunity is involved in the pathogenesis of both vitiligo vulgaris and palmoplantar pustulosis. It has been reported that interleukin-17 (IL-17) plays an important role in the pathogenesis of palmoplantar pustulosis, and that IL-17A expression is enhanced

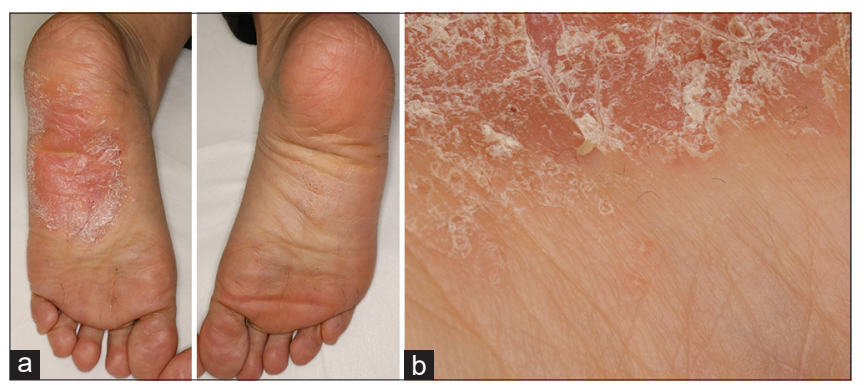

Figure 1: (a) Erythema, scales, and small vesicles on the left sole. (b) Small pustules were scattered close to the plantar erythema.

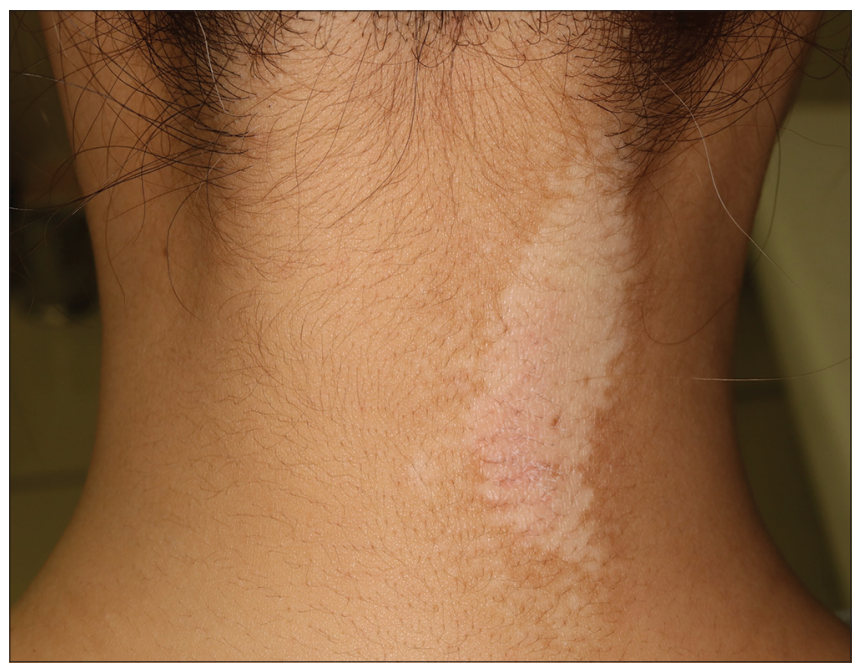

Figure 2: A depigmented macule surrounded by slight hyperpigmentation was also found on the right back side of the neck.

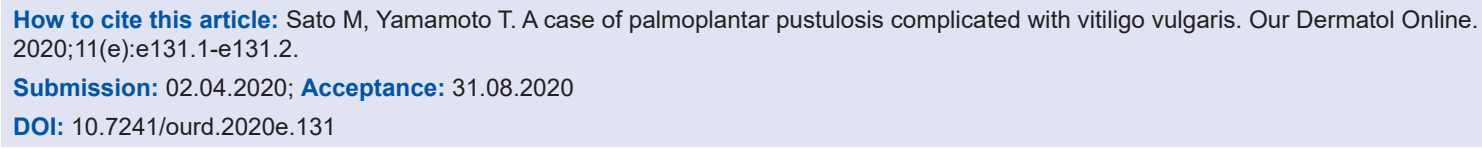




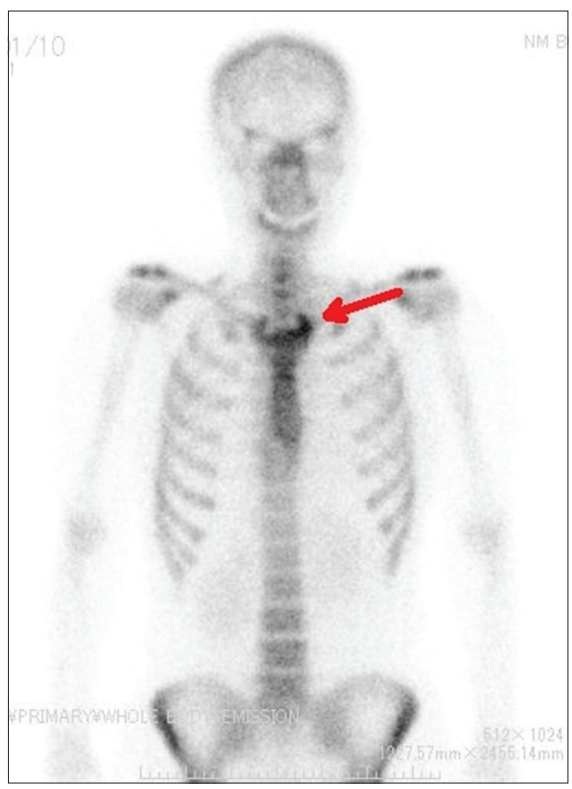

Figure 3: Bone scintigraphy showing slightly increased accumulation of technetium-99m in the left sternoclavicular joint.

in the lesional skin of palmoplantar pustulosis [1]. In addition, serum levels of IL-17 have been reported to be elevated in patients with palmoplantar pustulosis as compared with healthy individuals [2]. One aspect of the immune response affected by IL-17 is that keratinocytes in response to IL-17 promote the production of chemokine (C-X-C motif) ligand 1 (CXCL1), CXCL2, CXCL3 and CXCL8. Increased production of these chemokines may lead to neutrophil accumulation in the epidermis, leading to formation of pustules in palmoplantar pustulosis [1]. IL-17 is important also in the induction of palmoplantar pustulosis-associated osteoarthritis [3].

On the other hand, recent studies have demonstrated elevated systemic, local and cellular levels of IL-17 in vitiligo. Zhou et al. detected significantly higher serum IL-17 concentration in 45 patients with active vitiligo compared to 45 controls, using the enzyme-linked immunosorbent assay [4]. Furthermore, Wang et al. demonstrated higher numbers of cells positive for IL17A and IL-17A receptors in biopsy specimens of vitiligo lesions compared to skin specimens without lesions. IL17 was significantly correlated with autoimmune vitiligo, suggesting that IL-17 may be an integral factor in the progression and severity of vitiligo [5]. These reports suggest that IL-17 plays a common role in the etiology of both vitiligo vulgaris and palmoplantar pustulosis. Gill et al. reported that about $20 \%$ of vitiligo patients have an autoimmune disease, with thyroid disease being the most common [6]. In addition, it was reported that the rate of positive anti-thyroid antibody was significantly higher in palmoplantar pustulosis patients than in healthy controls [7]. The involvement of thyroid function in the pathology of vitiligo and palmoplantar pustulosis is unknown, but the pathogenesis of both may be related in some way. In the current case, anti-thyroid microsomal antibodies were slightly positive, and thus may be involved in the development of palmoplantar pustulosis and vitiligo. We here reported a case of palmoplantar pustulosis, pustulotic arthro-osteitis, and vitiligo vulgaris. To the best of our knowledge, there have not been reports of palmoplantar pustulosis and vitiligo existing concurrently in one patient; however, palmoplantar pustulosis may have been included in psoriasis, which occasionally co-exist with vitiligo.

\section{Consent}

The examination of the patient was conducted according to the Declaration of Helsinki principles.

The authors certify that they have obtained all appropriate patient consent forms. In the form the patient(s) has/have given his/her/ their consent for his/her/their images and other clinical information to be reported in the journal. The patients understand that their names and initials will not be published and due efforts will be made to conceal their identity, but anonymity cannot be guaranteed.

\section{REFERENCES}

1. Bissonnette R, Fuentes-Duculan J, Mashiko S, Li X, Bonifacio KM, Cueto I, et al. Palmoplanter pustular psoriasis (PPPP) is characterized by activation of the IL-17A pathway. J Dermatol Sci. 2017;85:20-6.

2. Murakami M, Hagforsen E, Morhenn V, Ishida-Yamamoto A, Iizuka H. Patients with palmoplantar pustulosis have increased IL17 and IL-22 levels both in the lesion and serum. Exp Dermatol. 2011;20:845-7.

3. Valeri M, Raffatellu M. Cytokines IL-17 and IL-22 in the host response to infection. Pathog Dis. 2016;74:ftw111.

4. Zhou L, Shi YL, Li K, Hamzavi I, Gao TW, Huggins R, et al. Increased circulating Th17 cells and elevated serum levels of TGFbeta and IL-21 are correlated with human non-segmental vitiligo development. Pigment Cell Melanoma Res. 2015;28:324-9.

5. Wang CQ, Cruz-Inigo AE, Fuentes-Duculan J, Moussai D, Gulati N, Sullivan-Whalen M, et al. Th17 cells and activated dendritic cells are increased in vitiligo lesions. PLoS One. 2011;6:e18907.

6. Gill L, Zarbo A, Isedeh P, Jacobsen G, Lim HW, Hamzavi I. Comorbid autoimmune diseases in patients with vitiligo: A crosssectional study. J Am Acad Dermatol. 2016;74:295-302.

7. Rosén K, Mobacken H, Nilsson LA. Increased prevalence of antithyroid antibodies and thyroid diseases in pustulosis palmoplantaris. Acta Derm Venereol. 1981;61:237-40.

Copyright by Mayu Sato, et al. This is an open access article distributed under the terms of the Creative Commons Attribution License, which permits unrestricted use, distribution, and reproduction in any medium, provided the original author and source are credited.

Source of Support: Nil, Conflict of Interest: None declared. 